\title{
Some New Approaches of the XXX Heisenberg Model of the Two-Magnon Sector
}

\author{
M. ŁABUZ ${ }^{a, *}$, J. MiLeWski ${ }^{b}$ AND T. LULEK ${ }^{c}$ \\ ${ }^{a}$ Department of Theoretical Physics, Faculty of Mathematics and Natural Sciences, University of Rzeszow, \\ Pigonia 1, 35-310 Rzeszów, Poland \\ ${ }^{b}$ Institute of Mathematics, Poznań University of Technology, Piotrowo 3A, 60-965 Poznań, Poland \\ ${ }^{c}$ Faculty of Physics, Adam Mickiewicz University, Umultowska 85, 61-614 Poznań, Poland and \\ East European State University in Przemyśl, Książąat Lubomirskich 6, 37-700 Przemyśl, Poland
}

XXX Heisenberg $s-1 / 2$ model has been examined in detail during last decades, however, recently one may find some new insights into that issue. Among several approaches describing the eigenproblem for the finite case, a close look into the structure of Bethe equations (BE) for the two-magnon sector case seems to be particularly interesting. BE enable us to evaluate parameters labeling eigenstates of a magnet, however to find appropriate sets of winding numbers, which parametrize BE, one has to apply the Inverse Bethe Ansatz method. On the other hand, one may choose a different - combinatoric approach - which also parametrizes Bethe eigenstates, with the use of rigging numbers describing string configurations. We present an idea of comparison of the concepts mentioned above for the particular case of two-spin deviations sector.

DOI: 10.12693/APhysPolA.133.444

PACS/topics: 75.10.Jm, 03.65.Fd, 03.67.Lx, 02.10.Ud, 02.10.De

\section{Introduction}

In the paper [1], it has been shown, that to solve an eigenproblem of two spin deviations sector for the $s-1 / 2$ Heisenberg isotropic, homogenous magnet ring, it is natural from the mathematical point of view to apply the approach by means of Chebyshev polynomials. In this paper, we intend to present explicit physical and topological parameters: riggings and winding numbers, for each of the exact eigenstate of the problem. One of the goals of the paper is a geometric interpretation of the Essler condition [2-4], which describes departure from the Bethe hypothesis for two spin deviations [5]. The paper is organized as follows. In Section II we introduce some notation of the eigenproblem of the two-deviation sector for arbitrary number of nodes $N \geqslant 4$, of the Heisenberg magnet. In particular, we present characteristic polynomial for this sector with a given total quasimomentum $k$ by means of suitable type of Chebyshev polynomial depending on the parity of $N$ and $k$. In Section III we recall Bethe equations for the two magnons case and present the solution by means of Inverse Bethe Ansatz method given in [6]. Bethe parameters are uniquely determined by quasimomentum $k$ and a new quantum number $m$ introduced in [1]. In Section IV we examine the difference of phases of Bethe parameters depending on $k$ and $m$ numbers. We introduce the notion of generalized bound and scattered states, and by means of these results we study their character. In Section $\mathrm{V}$ we parametrize winding numbers and rigged string configurations by $k$ and $m$

*corresponding author; e-mail: labuz@ur.edu.pl numbers. Finally, we show how winding numbers determine riggings of string configurations.

\section{Preliminaries}

In this section we give some general information concerning the problem being discussed, and recall results from [1]. Let $\mathcal{H}$ be the Hilbert space of the linear span over complex numbers on the set

$$
Q=\left\{\boldsymbol{j}=\left(j_{1}, j_{2}\right): 1 \leqslant j_{1}<j_{2} \leqslant N\right\}
$$

of states for $r=2$ spin deviations on the ring of $N$ nodes with a given natural scalar product. The action of the Heisenberg Hamiltonian $\hat{H}$ in the space $\mathcal{H}$ is given by

$$
\hat{H}|\boldsymbol{j}\rangle=\sum_{\mathbf{j}^{\prime} \in Q(j)}\left(\left|\boldsymbol{j}^{\prime}\right\rangle-|\boldsymbol{j}\rangle\right)+4|\boldsymbol{j}\rangle,
$$

where $|\boldsymbol{j}\rangle=\left|j_{1}, j_{2}\right\rangle$ denotes a state, with $j_{1}$ and $j_{2}$ indicating positions of Bethe pseudoparticles on a chain consisting of $N$ nodes, and $Q(\boldsymbol{j})$ is the set of all nearest neighbours of $\boldsymbol{j}[1]$. The summand $4|\boldsymbol{j}\rangle$ has been added for some convenience to shift the standard energy level, in order to simplify further calculations.

This Hamiltonian for any number of nodes $N$ and twomagnon sector can be introduced as the sum of blocks of Hamiltonians depending on values of quasimomenta $k$ :

$$
\mathcal{H}=\bigoplus_{k \in B} \mathcal{H}^{k}, \quad \hat{H}=\bigoplus_{k \in B} \hat{H}^{k} .
$$

Then, appropriate characteristic polynomials can be written as:

$$
P_{N}^{k}(x)=h^{M} \Phi_{N, M}^{k}(-u)+2 h^{M-1} \Phi_{N, M-1}^{k}(-u),
$$

where $h=|c|=|d|$ is the modulus of the hybridisation pameter, $u=x / 2 h$ and $M=\left[\frac{N}{2}\right]$ is the integer part of $\frac{N}{2}$.

Now, one introduces the function $\Phi_{N, n}^{k}$ depending on $N$ and $k$, such that: 


$$
\Phi_{N, n}^{k}= \begin{cases}2 T_{n}, & \text { for } N \text { even, } k \text { even, } \\ U_{n-1} / h, & \text { for } N \text { even, } k \text { odd, } \\ W_{n}, & \text { for } N \text { odd, } k \text { even, } \\ V_{n}, & \text { for } N \text { odd, } k \text { odd, }\end{cases}
$$

where $T_{n}, U_{n}, V_{n}, W_{n}$ denote the $n$-th Chebyshev polynomials of the suitable kind. For more details see [1].

\section{Bethe parameters}

Bethe equations may be introduced in the form [5]:

$$
\begin{aligned}
& N p_{1}=2 \pi n_{1}+\phi, \quad N p_{2}=2 \pi n_{2}-\phi, \\
& 2 \cot \frac{\phi}{2}=\cot \frac{p_{1}}{2}-\cot \frac{p_{2}}{2},
\end{aligned}
$$

with $p_{1}$ and $p_{2}$ denoting pseudomomenta, $\phi \in[0,2 \pi$ ) (for $k>0$, which we condider from now on, if not stated otherwise) describes phases of interacting pseudoparticles, and $\left(n_{1}, n_{2}\right)$ is a set of winding numbers, which parametrize Bethe equations.

Pseudomomenta $p_{1}$ and $p_{2}$ satisfy

$$
p_{1,2}=\frac{k \pi}{N} \pm \theta, \quad \theta \in[0, \pi]
$$

and from (8) we have the following equality:

$$
p_{1}+p_{2}=\frac{2 \pi k}{N} .
$$

Notice also that (9) describes conservation of pseudomomentum.

One can use Bethe parameters in the form of phases $(a, b)$, related with pseudomomenta by $a=\mathrm{e}^{-\mathrm{i} p_{1}}, b=$ $\mathrm{e}^{-\mathrm{i} p_{2}}$. In [6] a method of solving the problem of two deviation states was developed in detail, and relies on the use of the Inverse Bethe Ansatz (IBA) introduced therein. The main idea is to apply energy $E$ and total quasimomentum $k$ as known quantities, and evaluate portions of phase $(a, b)$ which parametrize the Heisenberg eigenstates. Eigenstates of the problem being discussed are labeled by two quatum numbers: quasimomentum $k$, and a new one $-m$, which depend on $N$ and parity of $k, m=0, \ldots, M(N, k)-1$, with $M(N, k)=M-r_{N, k}$, where

$$
r_{N, k}=\left\{\begin{array}{l}
1 \text { for } k \text { odd, } N \text { even, } \\
0 \text { otherwise. }
\end{array}\right.
$$

As a matter of fact IBA corresponds to the conservation laws of the total quasimomenta and energy, respectively:

$$
\begin{aligned}
& a b=\omega^{-k}, \omega=\mathrm{e}^{2 \pi \mathrm{i} / N}, \\
& a+a^{-1}+b+b^{-1}=E_{k, m},
\end{aligned}
$$

hence parameters $a$ and $b$ are roots of the quadratic equation

$$
\left(1+\omega^{k}\right) x^{2}-E_{k, m} x+\left(1+\omega^{-k}\right)=0 .
$$

\section{A determination of phase $\theta$}

In the paper [1], it has been shown, that portions of phase $a, b$ can be presented in a concise form

$$
a, b=\mathrm{e}^{-\mathrm{i} \frac{k \pi}{N}} \mathrm{e}^{ \pm \mathrm{i} \theta_{k, m}} .
$$

For real solution of $\theta$, in the case of $k$ even, one gets the estimation for $\theta_{k, m} \in\left(\frac{2 m+1}{N-2} \pi, \frac{2 m+2}{N-1} \pi\right)$, which satisfies the following equation

$$
\frac{\cos \frac{(N-2) \theta}{2}}{\cos \frac{N \theta}{2}}=\cos \frac{\pi k}{N},
$$

while for $k$ odd, one gets $\theta_{k, m} \in\left(\frac{2 m}{N-2} \pi, \frac{2 m+1}{N-1} \pi\right)$, and $\theta_{k, m}$ satisfies

$$
\frac{\sin \frac{(N-2) \theta}{2}}{\sin \frac{N \theta}{2}}=\cos \frac{\pi k}{N} .
$$

Analogously, for imaginary $\theta$, which at most occurs for $m=0$, one gets:

$$
\frac{\sinh \frac{(N-2) \eta}{2}}{\sinh \frac{N \eta}{2}}=\cos \frac{\pi k}{N}, \quad \text { for } k \text { odd },
$$

and

$$
\frac{\cosh \frac{(N-2) \eta}{2}}{\cosh \left(\frac{N \eta}{2}\right)}=\cos \frac{\pi k}{N} \quad \text { for } k \text { even, }
$$

where

$$
\theta_{k, 0}=\mathrm{i} \eta, \quad \eta>0 .
$$

Pseudomomenta of Bethe pseudoparticles for bound states are complex conjugate, and take the following form:

$$
p_{1,2}=\frac{k \pi}{N} \pm \theta_{0}=\frac{k \pi}{N} \pm \mathrm{i} \eta, \quad p_{1}, p_{2} \bmod 2 \pi .
$$

It is worth to recall, that for an arbitrary value of $k$, for the case $m>0$, the solution of $\theta_{k, m}$ is real, while for $m=0$, in most cases, $\theta_{k, 0}$ is imaginary. For that reason, for $m=0$ we call eigenstates generalized bound states (GBS), and for $m \neq 0$ - generalized scattered states (GSS). In most cases GBS is a bound state, and GSS - a scattered state. However, there are some exceptions:

A1. $k=0, m=0$, GBS is the second descendant of the vacuum,

A2. $k= \pm 1, m=0$, GBS is the first descendant of a suitable one-magnon state,

A3. $|k|>1, m=p_{k}$, where $p_{k}:=\left[\frac{|k|}{2}\right]$, GSS is the first descendant of a suitable one-magnon state,

A4. $|k|>1$ odd, for an $N$ large enough [2] (corresponding to $k)$, GBS $(m=0)$ appear to be scattered ones.

\section{Winding numbers}

In the case of real solutions of $\theta$, winding numbers satisfy the following relations:

$$
\begin{aligned}
& n_{1}=p_{k}-m, \quad n_{2}=p_{k}+m+r_{k} ; \\
& \quad m=0,1, \ldots, p_{k} \\
& n_{1}=p_{k}+m+r_{k}, \quad n_{2}=p_{k}+m+r_{k} ; \\
& \quad m=p_{k}+1, \ldots, M(N, k)-1,
\end{aligned}
$$

where $r_{N, k}$ is defined in (10), $p_{k}$ is given in A3, and 


$$
r_{k}=\left\{\begin{array}{l}
1 \text { for } k \text { odd, } \\
0 \text { for } k \text { even. }
\end{array}\right.
$$

For the case $m=p$, a state is a descendant arising from the space with a lower weight $r^{\prime}$. For $p>0$, a state with $m=0$ corresponds to a two-string, which means it is bound or scattered, depending on numbers $N, k$, according to the Essler considerations [2], what is connected with the A4 exception.

It is worth to observe that for a descendant $\left(m=p_{k}\right)$ the winding number $n_{1}=0$. Further, for the complex case of solution of $\theta$ one obtains

$$
\begin{aligned}
& k \pi+\mathrm{i} N \eta=2 \pi n_{1}+\phi \bmod 2 \pi, \\
& k \pi-\mathrm{i} N \eta=2 \pi n_{2}-\phi \bmod 2 \pi,
\end{aligned}
$$

with $\operatorname{Re} \phi \in[0,2 \pi), \operatorname{Im} \phi=N \eta$.

Then, depending on parity of $k$, one gets:

$$
\begin{array}{ll}
n_{1,2}=\frac{k}{2}, \phi=i N \eta, & \text { for } k \text { even, } \\
n_{1,2}=\frac{k \mp 1}{2}, \phi=\pi+i N \eta, & \text { for } k \text { odd. }
\end{array}
$$

Condition which enables one to choose riggings properly is as follows $[7,8]$ :

$$
0 \leqslant L \leqslant P_{l} \text {, }
$$

with $P_{l}$ being a maximum rigging value given by the following formula

$$
P_{l}=N-2 Q_{l}
$$

where $Q_{l}$ denotes the number of boxes in the first $l$ columns of the Young diagram of a string configuration $\nu$.

The relation of riggings to winding numbers, in the two-string case is the following

$$
L=n_{1}+n_{2}-2 \text {, }
$$

whereas for the case of two one-strings $\left(n_{2} \geqslant n_{1}+2\right)$ we have

$$
L_{2}=n_{1}-1, L_{1}=n_{2}-3 .
$$

\section{Conclusions}

In our paper we have examined the two-deviation sector of the Heisenberg magnet ring. We have shown, that eigenstates of the problem are labeled by quantum numbers: total quasimomentum $k$ and the number $m$, which determines generalized type of states, scattered or bound ones. This generalization corresponds to the nature of a state, with exceptions given by rules A1-A4 in Section 4. In particular, it is worth to observe, that winding numbers are related with riggings describing combinatiorial classification of the problem $((26),(27))$. Combinatorial approach and analytical solution give the same exceptions A1-A3. However, the Essler exception A4 for GBS leads to analytical scattered states (pseudomomenta are real), while from the combinatorial point of view solutions are classified as bound states $\left(n_{1}=n_{2}+1\right.$, for this case $r_{k}=1$ ).

\section{References}

[1] J. Milewski: Rep. on Math. Phys. 70, 345 (2012).

[2] F.H.L. Essler, V.E. Korepin, K. Schoutens, J. Phys. A 25, 4115 (1992)

[3] W.J. Caspers, M. Łabuz, A. Wal, M. Kuźma, T. Lulek, J. Phys. A: Math. Gen. 36, 5369 (2003).

[4] W.J. Caspers, A. Wal, M. Łabuz, M. Kuźma, T. Lulek: J. Math. Phys. 45, 391 (2004).

[5] H. Bethe: Z. Physik 71, 205 (1931) (in German; English translation in: D.C. Mattis, The Many-Body Problem, World Sci., Singapore 1993, p. 689.

[6] J. Milewski, G. Banaszak, T. Lulek, M. Labuz, Physica B 406, 520 (2011).

[7] T. Lulek: Banach Center Publications 78, 231 (2007).

[8] S. Dasmahapatra, O. Foda: Int. J. Mod. Phys. A 38, 1041 (1997) 